\title{
GESTIÓN CULTURAL Y PATRIMONIO EDIFICADO EN UN CONTEXTO URBANO. ESTUDIO DE CASO: “STTIO ARQUEOLÓGICO HUACA HUANTILLE”. LIMA-PERÚ [1982-2012] ${ }^{[*]}$
}

\section{CULTURAL MANAGEMENT AND BUILT HERITAGE IN AN URBAN CONTEXT. CASE STUDY: "HUACA HUANTILLE ARCHAEOLOGICAL SITE". LIMA-PERU [1982-2012]}

\author{
EVELYN CENTURIÓN CANCINO ${ }^{(x+)}$ \\ (D) https://orcid.org/0000-0003-1320-8444 \\ evelyn_gc6@hotmail.com \\ Universidad Nacional Mayor de San Marcos (Perú) \\ Fecha de recepción: 22 de abril de 2017 \\ Fecha de aprobación: 9 de febrero de 2020
}

\section{RESUMEN}

Por muchas décadas, los sitios arqueológicos del entorno urbano de Lima han sufrido constantemente ocupación ilegal, alteración, depredación, excavaciones ilícitas, entre otros estropicios cometidos contra una parte de nuestra memoria histórica que es fundamento vital para la identidad cultural de la ciudadanía peruana. Precisamente, el presente artículo está referido a uno de estos casos, al estudio desarrollado en Huaca Huantille, sitio arqueológico ubicado en el distrito de Magdalena del Mar, donde se han realizado denodados esfuerzos en pro de su recuperación, investigación y conservación como patrimonio cultural en un entorno urbano y que ahora nos plantea la urgencia de una nueva propuesta de gestión para lograr su revaloración cultural y puesta en valor definitiva.

\section{PALABRAS CLAVE}

Evaluación; Patrimonio arqueológico edificado; entorno urbano

\begin{abstract}
For many decades, the archeological sites in the urban environment of Lima have suffered constant damages, from its illegal occupation, alteration, predation, illicit digging, among others; being most of them part of the historical memory and training of the citizens in their cultural identity, each time archeological information is detached from them, which should be diffused in order to strengthen cultural policies promoted by the State to ensure its protection. It is in this regard this article comprises a case study of the archeological site Huaca Huantille, located in the district of Magdalena del Mar, Lima; where strenuous efforts are made for its restoration, investigation and conservation as built cultural heritage that is circumscribed within an urban environment, and in which it is urgent that a new management proposal is planned for its use and advantage as a resource and source of knowledge for the citizen; whose main element is the environment where it is circumscribed and a certain social group with different traditions, beliefs and principles concerning itself has been currently consolidated.
\end{abstract}

\section{KEYWORDS}

Evaluation; Built archeological heritage; urban environment

${ }^{*}$ ) El presente artículo es producto del trabajo de investigación realizado para el curso de Diagnóstico y examinación del Patrimonio Cultural a cargo del MSc. Arq. José Hayakawa Casas, del segundo ciclo de la Maestría de Gestión del Patrimonio Cultural, de la Unidad de Posgrado de la Facultad de Ciencias Sociales de la Universidad Nacional Mayor de San Marcos, en el año 2015.

(**) Licenciada en Arqueología por la Universidad Nacional Mayor de San Marcos, egresada de la Maestría en Gestión de Patrimonio Cultural por la Universidad Nacional Mayor de San Marcos, con estudios en Gestión Pública y actual Directora de la Dirección de Recuperaciones de la Dirección General de Defensa del Patrimonio Cultural del Ministerio de Cultura del Perú. 
devenir Vol. 7, Nº13, ENERO - JUNIO 2020, PP. 103-120 - EsTUDIOS I ISSN 2312-7562 | E-ISSN 2616-4949

UNIVERSIDAD NACIONAL DE INGENIERÍA, LIMA

doi: https://doi.org/10.21754/devenir.v7i13.927

\section{Antecedentes}

\section{Introducción}

Este estudio aborda la revisión y evaluación de las acciones que se realizaron para la recuperación, conservación, puesta en valor e investigación de la Huaca Huantille, sitio arqueológico ubicado en el distrito de Magdalena del Mar, de la ciudad de Lima. Se refiere también a la importancia histórica, informativa, social y económica que la recuperación de este patrimonio ha propiciado.

Es fundamental que se haya tomado en cuenta la opinión de la población circundante al momento de proponer nuevas acciones de intervención para su protección, conservación y difusión, resaltando algunos aspectos positivos como la ubicación del sitio arqueológico cerca de un grupo social y las condiciones actuales de recuperación y conservación que posee, para que este patrimonio no solo sea valorado sino puesto a disposición del público, y para que las acciones que se llevaron orientadas a su puesta en valor, no queden en el olvido. Para Ballart, Fullola \& Petit, (1996:215), se entiende por valor, a una cualidad añadida que los individuos atribuyen a ciertos objetos culturales, y que los hacen merecedores de aprecio, esto en función a diferentes referencias que varían dependiendo del grupo social, la contemporaneidad entre otros.

Por otro lado, fue necesario revisar y evaluar la documentación bibliográfica y las primeras descripciones como antecedentes para su declaratoria como Patrimonio Cultural de la Nación en el año 2001, y también los trabajos profesionales realizados entre los años 2006-2011 que permitieron obtener importante información arqueológica.

La metodología desarrollada -tipo cualitativa y cuantitativa- consistió en identificar a través de la observación el estado de conservación en que se encontraba este patrimonio cultural debido a los diferentes agentes de deterioro (naturales y antrópicos). Fue también importante conocer la percepción de la población circundante respecto al sitio arqueológico, a su valor y uso actual, a través del recojo de información por medio de entrevistas y encuestas para su posterior análisis y tabulación.

Además, esta evaluación permitió conocer cuáles son los valores, intereses y significados que el entorno social aledaño le otorga al sitio, y lo que la autoridad local propone respecto a la política participativa y a su utilidad actual, como un instrumento que oriente o encamine una determinada estrategia para el uso público de este patrimonio, permitiendo su sostenibilidad y preservación en el tiempo.

Finalmente, la propuesta de gestión para el sitio arqueológico Huaca Huantille, debe partir según lo que refiere Ladrón de Guevara (2009), de la idea de patrimonio como un sistema abierto con componentes materiales e inmateriales y que resalte la importancia del rol de la población aledaña en la evaluación de las condiciones actuales y en el uso que se le viene dando a este patrimonio.

\section{Antecedentes, reconocimiento, intervención y proyectos arqueológicos en Huaca Huantille (1892-2006)}

Es necesario conocer la importancia de este patrimonio edificado y sus primeras interpretaciones que se evidencian en las escasas referencias bibliográficas, intervenciones e investigaciones realizadas. Una de las primeras descripciones que nos aproximan a entender la inmensidad que tuvo este sitio arqueológico y su importancia, son las referencias que describen a Huaca Huantille de la siguiente manera:

Bandelier (1892) elabora un plano titulado: "Magdalena del Mar" en donde"...aparecen más de una docena de montículos de los cuales sobresale uno que consiste en dos pirámides escalonadas con rampas de gran tamaño y adosadas que se orientan 
hacia el Este, la pirámide doble corresponde a la actual Huaca Huantille o Magdalena...este edificio debió corresponder al templo de Magdalena que describe Squier" (1973:83-85).

De acuerdo a las prospecciones realizadas al sitio desde la década de los cuarenta, aparecen algunas de las publicaciones elaboradas por viajeros y arqueólogos que describen de manera ligera algunos recintos de este monumento, podemos citar algunos como Middendorf, (1984) "si continuamos nuestro paseo desde la Magdalena, encontraremos una Fortaleza que por su gran extensión se distingue, de todas las demás de esta región. Se trata de la huaca San Miguel, llamada así por el nombre de la hacienda vecina" (Roger 2012:11)

Por otro lado, Hutchinson (1873), refiere en relación a su ubicación y dimensiones, que alrededor de dos millas al sur del último gran fuerte mencionado, y en línea paralela con éste, como saludos al mar, encontramos otra estructura similar, probablemente un poco más espaciosa, y con un número más grande de compartimientos, o divisiones por muro sobre la cima, llamada San Miguel, creo que estas son las ruinas de la antigua fortaleza de Huantille, tiene cerca de 170 yardas de longitud por 168 a 170 de anchura, y es una enorme estructura de cerca de 90 pies de alto.

Cabe señalar, que el padre de la arqueológica peruana, Julio C. Tello (1999), ya describe este sitio como un cementerio que posee fardos funerarios, dentro del área de la misma waka, y comparada con otros complejos arqueológicos como Mateo Salado, debido a su magnitud, importancia y funcionalidad, que tenían en comparación con los monumentos del valle Rímac

A pesar de la vigencia durante los años 1936-1944, de la Ley de Salvaguarda del Patrimonio Cultural $\mathrm{N}^{\circ} 663$, que incluía a los monumentos anteriores al virreinato que refiere Tello (1999), en ese entonces se había activado de manera alarmante el derrumbe de los sitios arqueológicos. Entre 1941y 1944, la Huaca Huantille fue explotada con fines industriales por Víctor Lisandro Proaño y Tomas Percívale, lo cual ocasionó la destrucción de sus estructuras y también del cementerio que contaba con una gran cantidad de fardos funerarios.

En ese sentido, y a pesar de las constantes afectaciones que sufrió este monumento arqueológico, Huaca Huntille, de acuerdo a lo señalado por Guillen (2007), fue la más importante edificación de un grupo de monumentos conocidos en la actualidad con el nombre de Grupo Huantille; entre las edificaciones que se hallaron en la década del 40 fueron demolidas Huaca I, Huaca II, Huaca III y Huaca IV que estaban ubicadas a pocos metros de Huantille.

Ravines (1985), refiere que la Junta Nacional de Vivienda ordenó que Huantille fuera recortada en el lado Sur para poder construir un mercado que actualmente se encuentra junto a este patrimonio. Y en año 1972, la alcaldía distrital propuso su demolición para construir un Centro Cívico (ver Figura 1).

Asimismo, sobre sus dimensiones y características arquitectónicas, Ravines (1985), señala que Huaca Huantille, es una gran estructura piramidal de 60 metros de largo, por 40 metros de ancho y $12 \mathrm{~m}$ de alto, constituida por tres terraplenes, sobre los que se levantan gruesos muros de tapia en cimientos de piedra. Corresponde a un edificio post Tiahuanaco, integrante de un gran centro urbano, actualmente desaparecido. Sobre su arquitectura, señala que están visibles los patios, callejones, canchas cerradas y abiertas, recintos rectangulares hacia el lado Sur y Oeste, además de varios accesos, escaleras enlucidas y pintadas de blanco, con la presencia de un espacio abierto o plaza central ubicado en la cima de la pirámide orientada hacia el norte, donde se aglutinan una serie de conjuntos de canchas cerradas, recintos y terrazas escalonadas a manera de contrafuertes; en tanto este conjunto se edificó

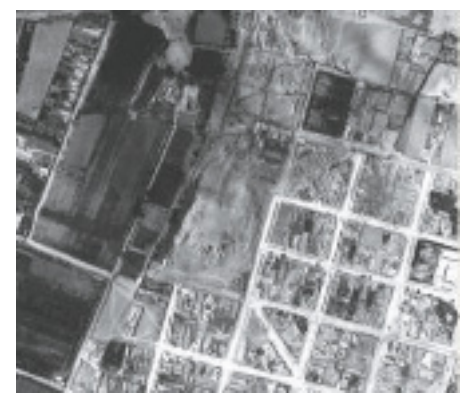

Figura 1. Fotografía aérea de 1946 que muestra las dimensiones que tenía entonces el sitio arqueológico. Tomada del Archivo del Servicio Aéreo Fotográfico Nacional, 1946. 


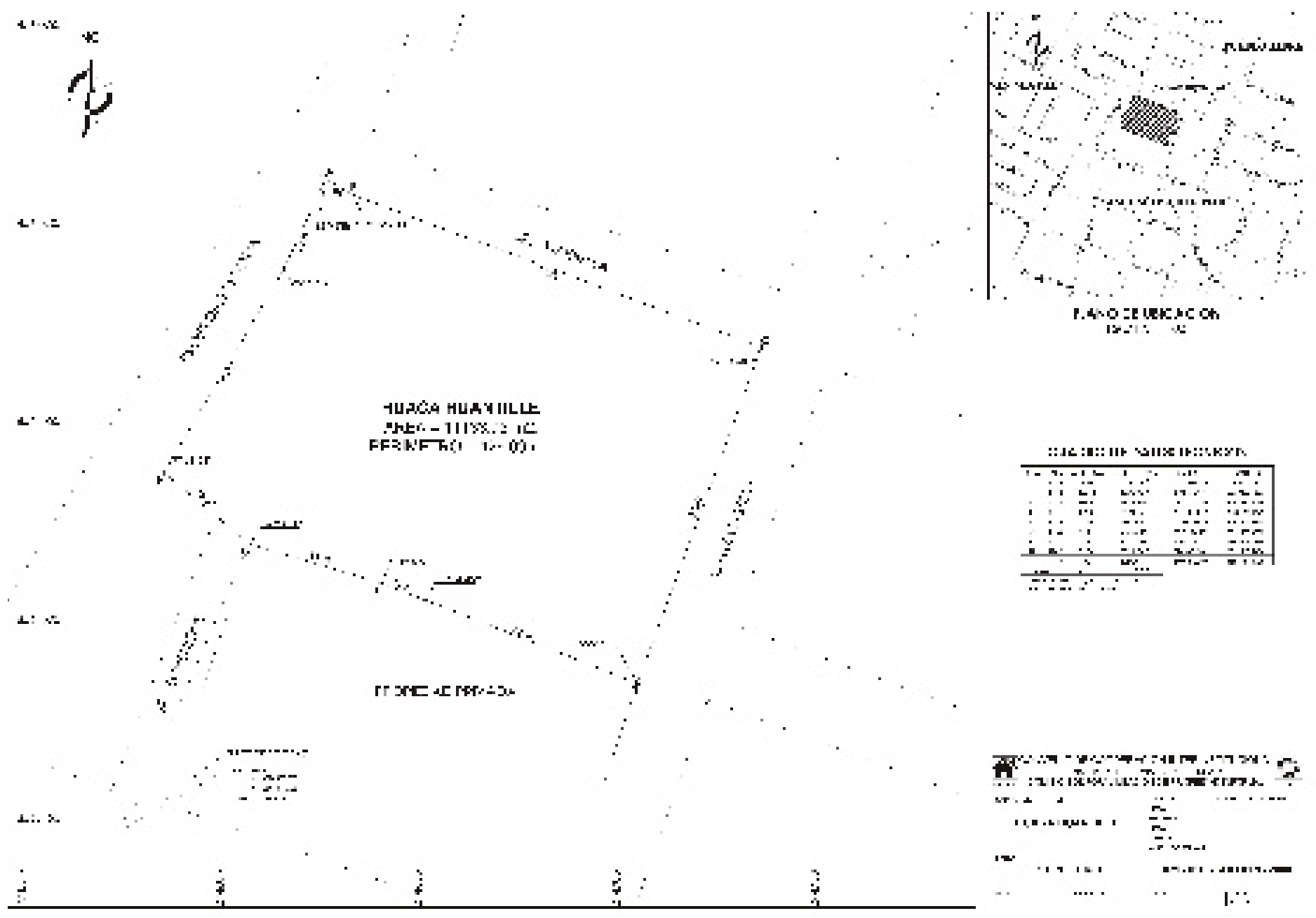

Figura 2. Plano Perimétrico de Huaca Huantille tomado de la Resolución Directoral Nacional $N^{\circ} 425 /$ INC, del año 2000. Editado por Estefanía Bernedo Ríos, 2019. a partir de muros de contención y muros superficiales en la parte superior, elaborados en tapia, hechos con alto porcentaje de arcilla, en menor proporción de arena, guijarros y fragmentos de cerámica, con relleno compuesto por cantos rodados y desmonte de demoliciones anteriores.

La Municipalidad Distrital de Magdalena del Mar, a inicios del año 2000, elaboró un Plan de Acción de Reasentamiento Huaca Huantille, definiéndola como una estructura piramidal de 60 metros de largo por 40 metros de ancho y 12 metros de alto, constituida por 3 terraplenes sobre los que se levantan gruesos muros de tapia sobre cimientos de piedra, en estas estructuras destacan patios, callejones, canchas cerradas y abiertas y recintos rectangulares, además de vanos de acceso, escaleras enlucidas y pintadas de blanco. Este plan de acción, fue elaborado con el propósito de dar a conocer la situación socio ambiental que había generado la ocupación ilegal de este sitio arqueológico, señalando la necesidad de reubicar a las familias allí asentadas a otro lugar.

Asimismo, en el año 2000, el entonces Instituto Nacional de Cultura (INC), hoy Ministerio de Cultura, Ilevó a cabo algunas acciones: se elaboró la Ficha Técnica del sitio arqueológico "Huaca Huantille" o "Echenique"; se revisaron tres inventarios: el Inventario y Catastro Arqueológico de los Valles Rimac y Santa Eulalia de 1975, el Inventario Monumental Arqueológico del Perú de 1985 (Ficha 66) y el Inventario del 
patrimonio Monumental Inmueble de Lima, Valles de Chillón, Rimac y Lurín de 1988 (Ficha 1018, éste último describe a la Huaca Huantille como: "Edificio público de característica piramidal, consta de tres niveles. La técnica constructiva es tapial, y se identifican patios, canchas, vanos de acceso, escaleras y muros enlucidos", con un área de 11,133.73 $\mathrm{m}^{2}$ y un perímetro de 426.09 m. (Ficha Técnica INC, Dirección General de Patrimonio Arqueológico, 2000:29)

Posteriormente mediante Resolución Directoral Nacional N 425/INC, de fecha 23 de mayo del 2001, se declara como Patrimonio Cultural de la Nación la Zona Arqueológica de "Huantille" o "Echenique", y se aprobó el Plano Perimétrico N021-INC-COFOPRI-2000 a Escala 1/500, la cual se consigna un área intangible de $1 \mathrm{Ha}+1,133.73$ $\mathrm{m}^{2}$ y un perímetro de 426.09 metros lineales, que rectifica el área señalada en las anteriores Resoluciones Ministeriales №732-87-ED y N0527-94-ED, que señalaban un perímetro de 410.56 metros lineales (ver Figura 2).

Como parte de los resultados de las investigaciones realizadas entre los años 2007 al 2011, se propone que el sitio arqueológico Huaca Huantille, corresponde a un Centro Ceremonial Administrativo de ocupación Yschma,

La presencia de una tinaja y su impronta en el piso, como parte de las evidencias, marcan una ubicación interesante, porque se ubican al centro de todos los recintos ceremoniales, restos domésticos (desperdicios de alimentos) asociados a la cerámica, creemos estar frente a espacios de usos múltiples, que servían tanto como habitaciones, como para hacer trabajos muy especializados dedicados al uso de la elite que debe haber ocupado estos recintos. (Guillén, 2012; p. 372)

En tanto, Huaca Huantille, es considera un centro administrativo ceremonial del curacazgo Lima, perteneciente al señorío Ychsma, que por sus características arquitectónicas corresponde a un edificio piramidal con una escalera principal como acceso hacia una plataforma central (de carácter ceremonial), en cuya cima se puede apreciar una serie de recintos principales y recintos secundarios, mucho de ellos, se muestran como salones amplios con baquetas, de acceso restringido y con presencia de pasadizos que hace presumir su uso de carácter público y doméstico; tal es el caso del área de producción y almacenamiento de bebidas, debido a la presencia de tinajas que describe Guillén (2012), además de una serie de recintos cuadrangulares, seguramente usados como áreas residenciales y depósitos.

Hoy en día se puede observar entre otros de sus componentes arquitectónicos, recintos menores, área de entierros, sector de tinajas y la presencia de frisos, actualmente cubiertos con triplay y plásticos debido a las condiciones climatológicas de la zona, éstos frisos muestran decoración geométrica y ornitomorfa, enlucidos blanco, crema y amarillo propios de la zona costera durante el Intermedio tardío (900-1450 d.C) con una plataforma central a la que se accede a través de una escalinata central y que conforman todo este conjunto piramidal que resalta entre los modernos edificios, producto de la expansión urbana de Magdalena (ver Figura 3).

La expansión urbana, fue un punto de quiebre para la preservación de todos los sitios arqueológicos de Lima, desde hace muchas décadas, cuyos contextos arqueológicos fueron ocupados ilegalmente, disturbados, alterados, convirtiéndose en sitios peligrosos y abandonados por el propio Estado, propicios para la depredación de la memoria histórica que dejaron nuestros antepasados. Se podría decir que a partir de los años 40 hasta la década de los 60, se agudizó el problema de la conservación de los monumentos.

En ese sentido, fue necesaria la reubicación de un grupo de aproximadamente 250 personas que hasta el año 2006 ocupaban este sitio arqueológico y vivían en condiciones precarias, dañando las estructuras de este patrimonio edificado. Fue entonces que por iniciativa de un grupo de funcionarios del entonces Instituto Nacional de

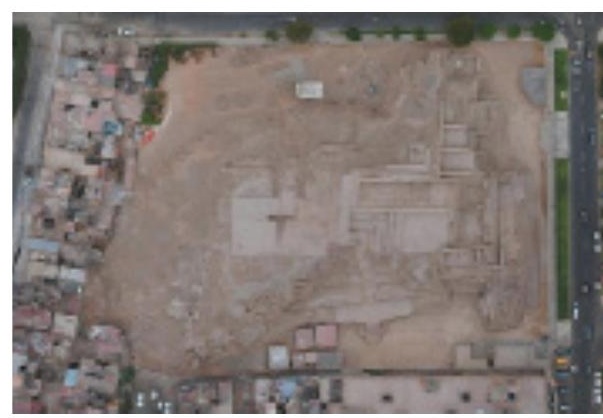

Figura 3. Vista aérea del Sitio arqueológico del 2018 y detalle. Recuperado de https://www.google.com/ maps/place/Huaca+Huantille, 2018. 
devenir Vol. 7, Nº13, ENERO - JUNIO 2020, PP. 103-120 - EstudIOS I ISSN 2312-7562 | E-ISSN 2616-4949

UNIVERSIDAD NACIONAL DE INGENIERÍA, LIMA

doi: https://doi.org/10.21754/devenir.v7i13.927

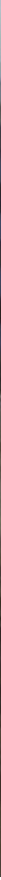

Figura 4. Vista del sitio arqueológico. Archivo personal de la autora en el marco de los trabajos realizados posteriormente a las dos temporadas del Proyecto, 2013.
Cultura (hoy Ministerio de Cultura), con el apoyo de la Municipalidad de Magdalena y las asociaciones de vecinos del distrito, entre otras autoridades de gobierno como COFOPRI, se logró su recuperación, retomando la tranquilidad del entorno y erradicando lo que se consideró por muchos años un foco de delincuencia e inseguridad.

Según la información publicada en el portal web de la Municipalidad de Magdalena, se hace referencia a la recuperación de este sitio arqueológico, y que tuvo como principal objetivo brindar a los precarios pobladores la posibilidad de tener una vida más digna, dotándolos de un fondo económico para su reubicación; logrando el desalojo de los ocupantes precarios de la forma más pacífica posible, sin enfrentamientos ni actos de violencia.

Posteriormente se consiguió que la población lo considerara suyo y que participara en las actividades culturales que se desarrollaban para la recuperación y posterior investigación del sitio arqueológico, concertando con las autoridades del gobierno central, instituciones públicas, privadas y la sociedad civil a fin de hacer viable este proyecto' (ver Figura 4). 


\section{Intervenciones en el sitio arqueológico (2006-2011)}

Es importante resaltar que la tranquilidad del entorno urbano del sitio, se recobró gracias a la solicitud y compromiso de la junta vecinal, al apoyo de la Municipalidad y las gestiones del entonces Instituto Nacional de Cultura, donde se proyectaron dos arduas temporadas de trabajo de investigación, conservación y puesta en valor, con el objetivo de recuperar las condiciones de conservación del mismo, y el rescate de importante información arqueológica, que permitió conocer algunos aspectos de la población prehispánica de entonces y sobre la funcionalidad del sitio y su enlace con la sociedad actual.

De las experiencias exitosas en recuperación de sitios arqueológicos del Patrimonio Cultural de la Nación, pocas veces se ha podido constituir una estrecha relación entre población y autoridades de gobierno para lograr un acuerdo o consenso no solo para la reubicación de los pobladores, sino para la recuperación de las condiciones de conservación del patrimonio arqueológico para su puesta en valor y uso de la ciudadanía en general, como se dio en este caso. Siendo Huaca Huantille, un ejemplo exitoso de recuperación de nuestro patrimonio cultural, con la reubicación de esa población precaria ilegalmente asentada hacía el distrito de Ventanilla, ha permitido posteriormente realizar un diagnóstico de emergencia con un adecuado manejo de del patrimonio edificado que actualmente se identifica con un grupo social en un entorno urbano de Lima.

Despues de las labores de erradicación de la población precaria que se asentó por casi 50 años en este sitio arqueológico, en el año 2007 se realizaron labores de limpieza y habilitación para dar inicio a la primera temporada de investigación, bajo la Dirección del Lic. Marco Guillén (2007), en amparo de la normativa vigente (RS 004200 ED) y en el marco de la Ley General del Patrimonio Cultural, se firmó el Convenio Específico de Cooperación Institucional suscrito entre la Instituto Nacional de Cultura y la Municipalidad de Magdalena del Mar, que permitió el financiamiento para su puesta en valor (ver Figura 5).

Los resultados de esta primera temporada, de acuerdo a lo señalado por Guillén $(2007)^{2}$, fueron insuficientes para obtener una comprensión cabal de la importancia de este patrimonio, debido a que las principales actividades realizadas en el sitio se centraron en la limpieza del área a investigar, retiro de desmonte, de material disturbado, entre otros. Y la actividad cultural no fue suficiente como en otros sitios del valle del Rimac. No obstante, respecto a la arquitectura, si se lograron de definir algunos recintos que caracterizaron a Huantille, como son los recintos de planta rectangular, muros de tapia, pisos a desnivel, vanos de acceso, banquetas, la escalera principal a la plaza central, y edificada por grandes muros de tapial de sección trapezoidal con bases que miden de 1.00 a 2.80, con la presencia de rellenos arquitectónicos construidos por fragmentos de cerámica, cantos rodados, o fragmentos de tapial vistos en los perfiles que se exponen actualmente.

Respecto a las evidencias halladas en su mayoría pertenecen a fragmentos de cerámica Yschma y presencia de material estilo Chancay de otros sitios, restos óseos, moluscos, entre otros. Es por ello, que la primera conclusión a la que llega este proyecto sobre la funcionalidad del sitio, es que corresponde a un centro de la importante sociedad Ychsma construido con grandes muros de tapia que permitieron darle la altura y dimensión actual.

Tal como hemos señalado, los resultados de esta investigación, como la evidencia de un patrón de pirámide con escalera central, la presencia de recintos públicos y privados diferentes entre sí, proponen que la Huaca Huantille fue un importante del

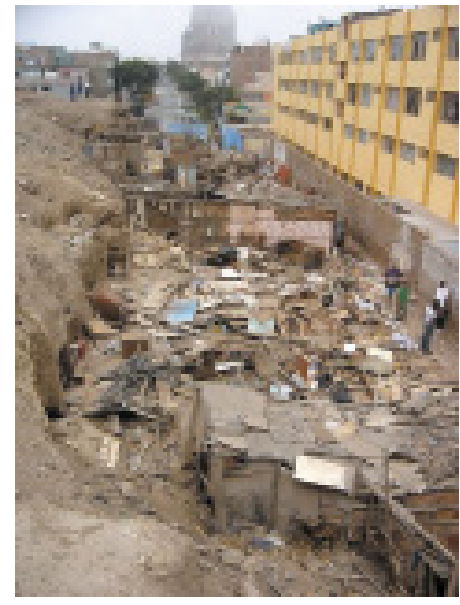

Figura 5. Vista del sitio arqueológico antes de sus intervenciones. Recuperado de Municipalidad de Magdalena, http://www.munimagdalena.com, 2017.

2. Informe final del Proyecto de Investigación, Conservación y Puesta en Valor de la Huaca Huantille (2007-2011) 


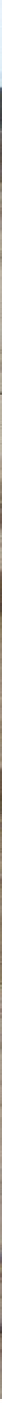

Figura 6. Intervenciones de conservación en el sitio. Archivo del Proyecto- Temporada 2013, 2013. centro administrativo ceremonial durante el intermedio (900-1432 d.C) en el Valle bajo del río Rimác. No obstante, aún quedan muchas investigaciones que puedan contrastar las propuestas vertidas por los investigadores de los sitios del valle bajo del río Rímac, durante el periodo de ocupación Yschma.

Los hallazgos realizados en la Huaca Huantille (Guillén, 2012), confirmaron una data de al menos tres periodos de ocupación o momentos constructivos que fueron encontrados disturbados, los dos momentos con un salón principal en la cima al cual se tenía acceso por escalinatas de dos periodos; el primer momento corresponde a la fachada más antigua que se le conoce al edificio con la presencia de una escalera delgada que fue enterrada por completo para dar espacio al segundo momento, el cual presenta una ampliación del salón principal asociado a una escalinata principal con mayores dimensiones y nuevas plataformas, finalmente el último momento es el abandono de la huaca con la presencia de corrales.

En el año 2011-2012, empezó la segunda temporada de intervención del Proyecto de Investigación, Conservación y Puesta en valor, cuyos resultados arrojaron mayores luces sobre la interpretación, ya que los trabajos se centraron en la excavación de los cuadrantes Noreste y Sureste del sitio, en plena cima del edificio. Gracias a estas intervenciones se pudo identificar otros recintos que contenían algunos restos domésticos de cerámica, de manufactura textil y también desperdicios de alimentos, hallazgos que brindaron más luces sobre la arquitectura del sitio, definiéndola como una pirámide aterrazada trunca con una escalinata central cuyo material de cons- 
trucción era básicamente una tapia al interior de la cual se podían observar algunos recintos menores de planta ortogonal con banquetas y vanos de acceso. Como centro administrativo ceremonial tuvo mayor actividad y presencia durante el Intermedio Tardío (900-1432 d.C) del valle bajo del río Rímac (ver Figura 6).

Posteriormente, durante las dos temporadas que duró dicho proyecto, los trabajos de conservación de las estructuras del sitio tuvieron prioridad, incluso desde sus inicios, con acciones de conservación preventiva y restauración. La interpretación preliminar de los elementos hallados en sus contextos funerarios, a pesar de las malas condiciones en que se encontraban, y las buenas iniciativas de gestión avizoraban lo que sería un gran reto para la recuperación de este Patrimonio Edificado.

En ese sentido, queda mucho por trabajar en cuanto a la investigación del material arqueológico procedente de ambas temporadas, y que, al término del último proyecto, no se ha difundido ni puesto en conocimiento de la ciudadanía. Tampoco se ha profundizado en la identificación de ocupaciones posteriores. Sin embargo, sería importante señalar las razones por las que en este corto periodo de intervención no se pudo exponer mayores interpretaciones arqueológicas sobre el sitio. Además, es necesario señalar que no se pudo contar con todas condiciones necesarias para llevar a cabo las acciones de conservación, ni la participación de la comunidad en las mismas por la falta de una adecuada difusión cultural con proyección a futuro que cuente con aliados estratégicos para su sostenibilidad.

\section{Estado post- intervención del sitio arqueológico}

Las acciones desarrolladas en Huantille en el marco de las dos temporadas de investigación y puesta en valor, han generado un espacio para la ejecución de actividades culturales y de integración con la comunidad del entorno. Y, si bien es cierto que no hubo continuidad luego de la culminación de los mismos, de alguna manera ha permitido su reconocimiento y valorización como patrimonio cultural.

Es en ese sentido, es importante mencionar que en el marco de la ejecución del proyecto de investigación y su puesta en valor, durante las dos temporadas mencionadas se impulsaron actividades y representaciones de tipo cultural que han generado una importante interacción social; por ejemplo, tuvo lugar la escenificación de la tradicional ceremonia del Inti Raymi o Fiesta del Sol, majestuosa puesta en escena con la participación de más de 60 actores y la asistencia de cientos de vecinos e invitados. "De esta manera se recreó una antigua ceremonia religiosa a través de diversas manifestaciones culturales como danzas y música". (Guillén, Temporadas 2011 y 2012) (ver Figura 7).

Actualmente, este sitio cuenta con las condiciones mínimas para el desarrollo de actividades de promoción y difusión cultural, tiene ambientes de material noble y concreto con servicios básicos (agua, desagüe y luz). Y cuenta también con un sistema de iluminación gracias a Plan COPESCO de MINCETUR en año 2012. Además, se le ha declarado Zona Intangible por ser Patrimonio Cultural de la Nación, con Resolución Directoral Nacional N²425/INC en el año 2001.

El entorno del sitio arqueológico está constituido por viviendas unifamiliares y multifamiliares, además de negocios locales; y actualmente no cuenta con una señalización adecuada debido a la falta de mantenimiento de todo el sitio, sus elementos de concreto se encuentran rajados o rotos con una estructura interna corroída. Asimismo, hay una vivienda, con un cerco perimétrico en mal estado de conservación, que presenta graves faltas de seguridad y que aún se mantiene al interior del polígono del sitio arqueológico porque nunca pudo ser reubicada (ver Figura 8). 


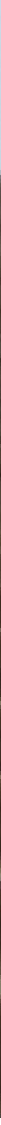

Figura 7. Reapertura de la Huaca Huantille a la visita de los vecinos. Archivo personal de la autora en el marco de los trabajos realizados posteriormente a las dos temporadas del Proyecto, 2013.

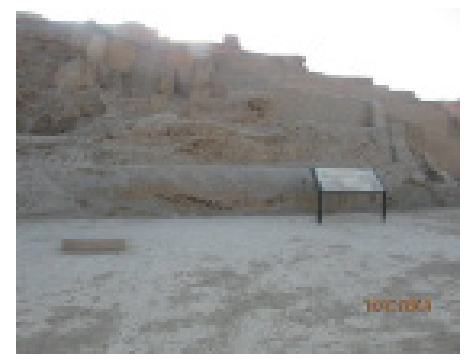

Figura 8. Vista de las condiciones actuales sitio arqueológico con un panel infográfico. Archivo personal de la autora, 2015.
Sin embargo, es importante identificar los valores que posee el sitio por ser generados en su contexto ambiental y social. Para Ballart\&Fullola\&Angel, (1996) el potencial de los bienes históricos como recurso ha de ser evaluado, en cualquier caso, a la luz de los contextos específicos, ya que el valor atribuible solo puede darse en situaciones reales y socialmente determinadas.

En el proceso de valoración de los recursos culturales, intervienen múltiples interlocutores con diferentes percepciones e intereses en torno al patrimonio que en algunas ocasiones suelen ser contrapuestas, por lo que se hace necesario identificar a los interesados como agentes de valoración en todas las comunidades (Ladrón de Guevara, 2009: 66).

Los valores identificados en este sitio arqueológico que podrían traer utilidad y beneficio a la población, son los siguientes:

A) Informativo y científico: Referentes al contenido de los resultados de las primeras interpretaciones e intervenciones realizadas en el Sitio durante los años 2007, 2011 y 2012, además de la información obtenida producto de las investigaciones realizadas y el potencial que tiene para seguir siendo intervenido y obtener mayores datos.

B) Valor histórico: La importancia que tenía el sitio como centro administrativo-ceremonial donde confluían diferentes poblaciones en el valle bajo del rio Rímac, de acuerdo a las primeras publicaciones y referencias sobre la importancia del mismo. 


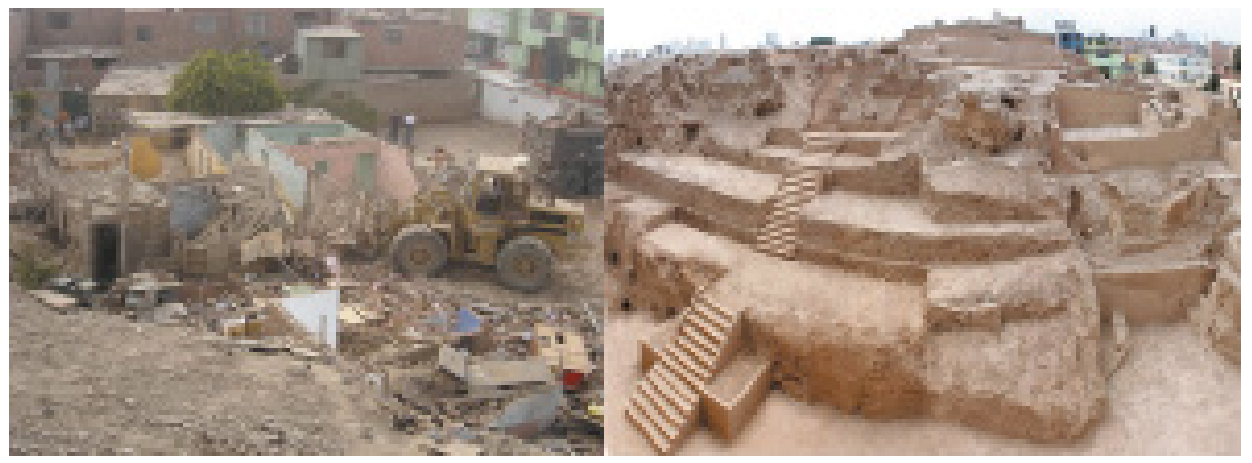

Figura 9. Condiciones anteriores y posteriores a la intervención del sitio arqueológico Huantille. Archivo del Proyecto arqueológico temporada. 2011-2012, 2012.

C) Valor social: La participación de la comunidad en actividades que se desarrollan en los espacios de uso social en el sitio.

D) Valor económico: Concepto por ingreso al sitio y otras actividades que se generen en el mismo,

Entre los aspectos positivos logrados posteriormente a la recuperación y la intervención del sitio podemos detallar lo siguiente:

- Infraestructura: cuenta con dos ambientes de material noble y de concreto (servicios higiénicos y servicios básicos (agua, desagüe y luz).

- Iluminación: Plan COPESCO, realizó un proyecto de iluminación nocturna, para el año 2012.

-Señalización: cuenta con paneles de señalización (aunque no se encuentran en buenas condiciones)

- Muro perimétrico: Sin embargo, las bases de cerco perimétrico en algunos sectores se viene erosionando rápidamente debido a la humedad y acciones vandálicas.

-Firma de un Convenio Marco Interinstitucional (actualmente caducado): se dio entre el Ministerio de Cultura y la Municipalidad de Magdalena en acuerdo con el cumplimiento de la Ley $\mathrm{N}^{\circ} 27971$, Ley Orgánica de Municipalidades y en coordinación con las instancias institucionales de competencia.

No obstante, existen algunos aspectos negativos que son importantes mencionar:

- Inexistente fuente de financiamiento (falta del incremento de la partida presupuestal por parte de la Municipalidad).

- Desconocimiento parcial por parte de la población respecto al sitio arqueológico.

- No hubo continuidad en los trabajos de investigación y puesta en valor, no hubo responsabilidad en el manejo del sitio a tiempo completo, ni proyección a otras acciones luego de las intervenciones realizadas.

Actualmente el sitio viene siendo administrado por la Municipalidad de Magdalena, y ya no cuenta con servicio de vigilancia las 24 horas para asegurar su protección; pocas son las actividades relacionadas a la gestión cultural y difusión del patrimonio arqueológico hacia la comunidad,con un acceso limitado (ver Figura 9). 
devenir Vol. 7, Nº13, ENERO - JUNIO 2020, PP. 103-120 - EsTUDIOS I ISSN 2312-7562 | E-ISSN 2616-4949

UNIVERSIDAD NACIONAL DE INGENIERÍA, LIMA

doi: https://doi.org/10.21754/devenir.v7i13.927

\section{Metodología y proceso de diagnóstico}

Entender la dinámica de este tipo de patrimonio edificado, el análisis e interpretación y la importancia de su conservación son fundamentales en el manejo y tratamiento adecuado del mismo, promoviendo su valor como reflejo de una manifestación cultural. Krebs \& Schimidt (1999), sostienen que a diferencia de los bienes naturales, los bienes del patrimonio cultural son recursos no renovables, y es allí donde radica la responsabilidad de asegurar la preservación de nuestra herencia cultural para la investigación y para el disfrute de generaciones futuras.

En ese sentido, es importante señalar que todas las propuestas hechas posteriormente a esta evaluación, recogen las perspectivas, inquietudes, expectativas e intereses de la ciudadanía, analizan las condiciones y características de este sitio arqueológico para que su gestión, conservación, protección y puesta en valor definitiva sean factibles.

El contexto ambiental y sociocultural es fundamental para la evaluación de un bien patrimonial, como señala Ladrón de Guevara \& Elizaga (2009:65). La contextualización territorial del recurso cultural es la base que permite comprender las dinámicas de conservación del sitio, conocer los componentes bióticos y abióticos del medio, como también considerar sus aspectos socioculturales y administrativos actuales y previsibles.

Es importante señalar, que en el sitio arqueológico Huaca Huantille, no se han efectuado diagnósticos con la misma profundidad; el diagnóstico realizado después de su recuperación en el año 2006, solo detalla las condiciones en que fue encontrado y propone los principales tratamientos para su conservación y la metodología para su posterior intervención. Entre tanto, se realizó un análisis preliminar de las estructuras expuestas que permitió identificar las condiciones de los bienes culturales hallados y los principales problemas para su preservación.

Debemos considerar que la gestión del patrimonio cultural se vierte en un proceso participativo construido en la cotidianeidad y de forma conjunta y abierta, a través de la interacción de diferentes actores que cooperan entre sí para disfrutar de ese patrimonio, y asegurar al mismo tiempo su transmisión a generaciones futuras. El patrimonio es un legado, y sólo tiene valor en la medida en que es reconocido y tiene significado en la vida de un individuo, grupo o comunidad, fortaleciendo su identidad (Monsalve; 2011:39).

En el presente caso, fue necesario realizar ciertas acciones de reconocimiento del entorno social, las perspectivas, intereses e inquietudes de la población, respecto a la identificación, valoración y protección de este sitio arqueológico. De acuerdo a lo que señala Ladrón de Guevara \& Elizaga (2009), los recursos patrimoniales se deben considerar como un sistema complejo y dinámico, caracterizado por la presencia de múltiples voces, formas de ser y estar en este mundo, la única manera de generar acciones perdurables y respetuosas con todos aquellos que se sienten vinculados a dichos recursos. Por eso, es importante que cualquier gestión que se pretenda realizar en el sitio debe estar orientada por los resultados obtenidos en los sondeos, entrevistas y encuestas realizados a la ciudadanía sobre la percepción y valoración del mismo.

El sondeo posterior a las intervenciones realizadas en el sitio, permitió obtener información cualitativa y de manera rápida, tomando una muestra de un segmento de la población circundante a Huaca Huantille. El objetico específico era conocer la percepción e identidad de la población que habita en el entorno y ha visitado el sitio arqueológico. saber si lo recuerdan como un espacio de interacción social. Los encuestados se mostraron a favor de su recuperación y del inicio de los trabajos de puesta en valor (ver Figura 10). 
Conocimientos y valoración de Huaca Huantille

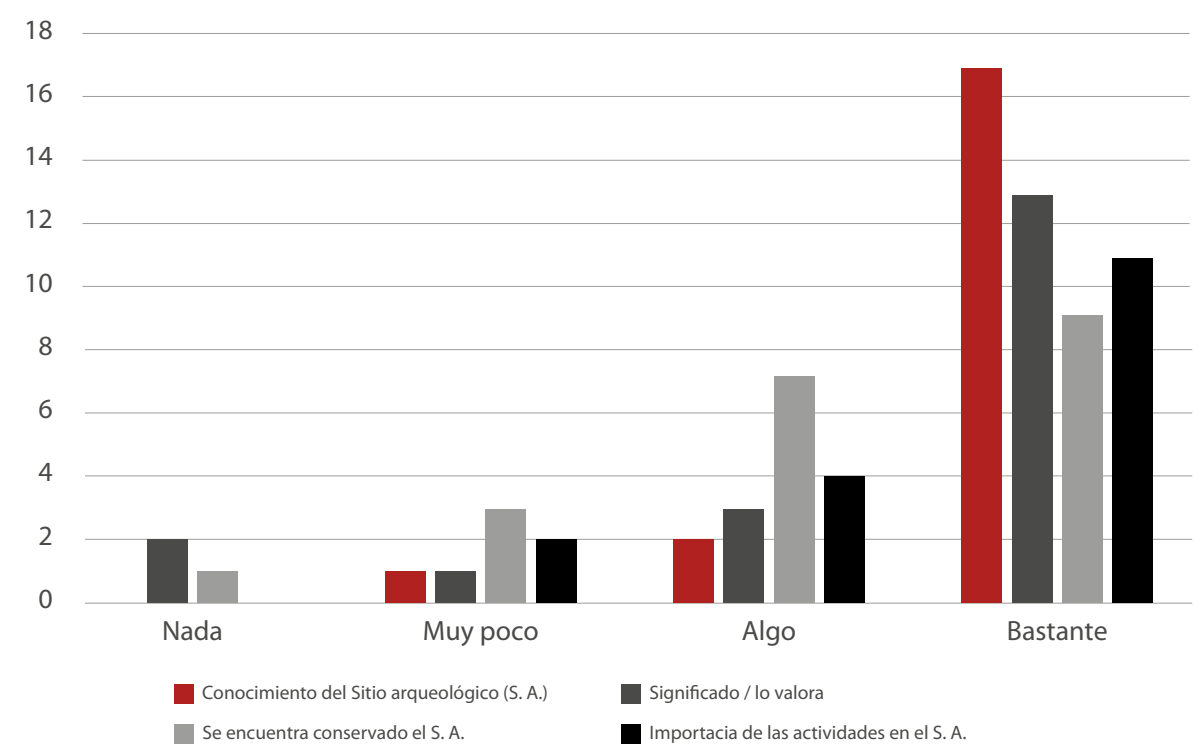

Figura 10. Conocimiento y valoración sobre el sitio arqueológico por parte de la población vecina. Elaboración propia, 2016.

El presente análisis no solo busca detallar los componentes arqueológicos del sitio y la importancia de conocer los resultados de las investigaciones realizadas, sino determinar por qué es necesario realizar un diagnóstico de la actual situación y el manejo que se le viene dando al mismo; Gracias a la difusión de la información arqueológica que se ha obtenido para conocimiento y uso de la población se podrá proponer mejores acciones para su revalorización. "Al tener en consideración que estos individuos, grupos y comunidades se expresan de forma diferente, caemos en la cuenta de que las expresiones tangibles e intangibles del patrimonio cultural son tan disímiles como son los individuos, grupos y comunidades. En tal sentido, no se puede hablar de modelos establecidos de gestión para cada ámbito del patrimonio cultural". (Monsalve; 2011:39).

En nuestro país existen varias zonas arqueológicas que están situadas en diferentes entornos, algunas en buen estado de conservación y otras en total estado de abandono por la escasa intervención directa del Estado. Para poder conservar nuestro patrimonio cultural necesitamos contar con la colaboración e interés de los ciudadanos que habitan en la zona adyacente, ya que ellos son los protagonistas de este bien patrimonial y de esta manera se hace necesario hacerlos partícipes en la defensa, protección y difusión, de manera que se sientan identificados con su patrimonio.

\section{Etapas de intervención realizadas}

Respecto al diagnóstico realizado en el mismo, es necesario exponer algunos alcances obtenidos y las limitaciones presentadas durante este proceso de evaluación. Los alcances han permitido conocer las condiciones en que se encontraba este patrimonio edificado, identificar los principales agentes de su deterioro, tales como la concentración de humedad, vientos, lluvias, radicación solar, shock térmico, por ejemplo la excesiva concentración de sales que se encontraron en el material arqueológico, la erosión de los muros, todo esto aunado a las consecuencias de la 
devenir Vol. 7, Nº13, ENERO - JUNIO 2020, PP. 103-120 - EsTUDIOS I ISSN 2312-7562 | E-ISSN 2616-4949

UNIVERSIDAD NACIONAL DE INGENIERÍA, LIMA

doi: https://doi.org/10.21754/devenir.v7i13.927

invasión que sufrió y que orginó la falta de estabilidad de sus estructuras y la reducción de sus espacios originales.

Entre las limitaciones presentadas durante este diagnóstico, es que sólo se desarrolló a grandes rasgos una propuesta para los procedimientos de conservación a fin de lograr la estabilidad definitiva de sus estructuras. Se tiene poca información sobre el tratamiento realizado a las estructuras, si bien se encuentran estables, no se tiene detalles específicos sobre los procedimientos efectuados.

De los criterios teóricos y específicos realizados en la evaluación, consideramos los siguientes:

- Revisión de información documental detallada en los informes finales acerca de las evaluaciones realizadas a este patrimonio edificado.

- La utilización de los principios básicos de asociación y contexto.

- Una fuente de información también son los hallazgos realizados en contexto, ya que han permitido obtener mayores detalles de la evaluación.

Las acciones contaron con la participación de la Municipalidad de Magdalena que proporcionó los recursos para su ejecución y, sin duda, el segmento de la población que ocupaba el sitio y que fue reubicada por este municipio, jugó un rol importante en la realización de este diagnóstico y también gracias a la cooperación de algunos vecinos que estaban interesados en la recuperación de este monumento arqueológico. (Marco Guillén, en comunicación personal).

Las condiciones negativas identificadas a partir del diagnóstico realizado, como se ha podido detallar, son las que se dieron a partir de la ocupación y alteración de los contextos arqueológicos.

La problemática radica en que la ocupación ilegal de más de cuatro décadas ha afectado considerablemente el monumento, dejando sobre expuestas estructuras, rellenos constructivos y el material arqueológico. Los perfiles estratigráficos exponen los rellenos constructivos originales que corren peligro de desmoronamiento por desestabilización estructural. Por tanto, el factor humano se ha convertido en el principal agente de destrucción y depredación sistemática del monumento. (Guillén, Diagnóstico integral del sitio arqueológico)

Sin duda, son muchas las causas que llevaron al deterioro parcial de este sitio arqueológico, pero la que se dío por agentes antrópicos produjo la alteración de los rellenos arqueológicos y el impacto directo sobre sus estructuras (tapial), que dio lugar a deslizamientos del material. “La alteración de la superficie arqueológica y del área de protección del monumento es tangible: destrucción ex profeso de estructuras arqueológicas y alteración de rellenos constructivos como componentes básicos de la estructura piramidal trunca y la alteración del entorno paisajístico" (Guillén, Diagnóstico integral del sitio arqueológico, 2006, p. 6).

Es importante destacar la ardua labor y entrega por la recuperación y protección del Patrimonio Cultural, realizado por el equipo de arqueólogos del entonces Instituto Nacional de Cultura, hoy Ministerio de Cultura, y el apoyo de la Municipalidad de Magdalena del Mar, desde el inicio de su temporada de investigación, conservación y puesta en valor en el año 2007. No está demás enfatizar en su gran labor porque las condiciones calamitosas y deplorables causadas por las constantes ocupaciones avizoraban un panorama imposible para su recuperación. Un verdadero reto que debería ser modelo de gestión para nuestro patrimonio cultural que aún persiste hasta nuestros días. 


\section{Propuesta}

Además de las acciones efectuadas para el conocimiento de las perspectivas, intereses e inquietudes de la población respecto a la identificación, valoración y protección del sitio arqueológico, durante este proceso de evaluación se han definido etapas, actores involucrados, problemáticas que surgieron durante ese proceso, los resultados obtenidos y algunos aspectos relevantes del caso. Así también es necesario conocer las condiciones en que se encontraba este patrimonio edificado y los factores de deterioro que han incidido negativamente en la conservación de este patrimonio son de diversa naturaleza como señalamos líneas arriba.

Se procedió con la revisión bibliográfica, específicamente de algunas publicaciones que describen ligeramente algunas características del sitio arqueológico (Ravines, Tello, Hutchison), toda vez que no contaban con antecedentes de investigación para incluir los resultados de la primera etapa de investigación en el sitio (2007-2011). Además se identificaron las acciones de destrucción originadas por el deterioro de las estructuras y por ende los contextos funerarios con la consiguiente alteración de la evidencia arqueológica.

La gestión que se propone para este tipo de patrimonio edificado, implica que se cumplan ciertos propósitos orientados al estudio, conservación, difusión y revaloración del patrimonio cultural, considerando lo que señala (Román, 2011, p. 8) “la gestión cultural es la administración de los recursos de una organización cultural, con el objetivo de ofrecer un producto o servicio que llegue al mayor número de público o de consumidores", esta última sostiene que "la propia necesidad de esta formación nace de estas instituciones culturales, más específicamente desde los museos, como espacios especializados en la salvaguarda y la difusión del patrimonio cultural, clave en la formación de gestores culturales".

El principal objetivo, es restablecer las actividades de difusión cultural en el sitio, desde una propuesta de promoción cultural, destinada a la ciudadanía general, a fin de generar la revaloración e importancia de este patrimonio cultural edificado, que actualmente está olvidado, a fin de poder generar y garantizar acciones para su salvaguarda a partir de generaciones futuras. Como refiere Monsalve (2011), un primer paso en la gestión del patrimonio es su identificación a través del registro, la catalogación y la construcción de inventarios, pues, es imposible poner en valor, difundir y sensibilizar sobre lo que no se conoce. Una vez identificadas aquellas expresiones tangibles e intangibles del patrimonio, que pretendemos salvaguardar por su valor cultural, social o económico, entonces podemos pensar cómo gestionarlas para lograr su salvaguarda y viabilidad.

Entendemos que no podrá ser una tarea fácil, ya que desconocemos la predisposición de los diferentes actores comprometidos y la voluntad política con la que actualmente podría contar este sitio arqueológico; si bien es importante considerar la evaluación realizada en base a la percepción actual de la población ubicada en el entorno y con la cual ya se ha trabajado, hay que tener en cuenta que toda sociedad puede ser pasible de los cambios de la globalización que desvirtúan su identidad cultural; razón por la cual es necesario restablecer y reforzar el sentido de identificación y revalorización de este patrimonio cultural.

\section{Conclusiones}

De la interpretación de los resultados obtenidos durante el proceso de evaluación, es importante resaltar algunos alcances para una propuesta de gestión cultural integral y sostenible que permita que se ejecuten acciones dinámicas y permanentes, empezando con el sector de la población que actualmente se ubica en los alrededores del sitio, y que de cierta forma, actualmente se siente vinculado e identificado con su patrimonio arqueológico. 
devenir Vol. 7, Nº13, ENERO - JUNIO 2020, PP. 103-120 - EsTUDIOS I ISSN 2312-7562 | E-ISSN 2616-4949

UNIVERSIDAD NACIONAL DE INGENIERÍA, LIMA

doi: https://doi.org/10.21754/devenir.v7i13.927

La problemática, como resultado de esta evaluación, determinó que la ocupación ilegal de hace varias décadas no solo afectó a las estructuras del monumento, sino también las evidencias arqueológicas, toda vez que los contextos se hallaron disturbados y alterados al interior de las estructuras y rellenos constructivos. Lo cual no ha permitido una aproximación y conocimiento integral sobre el sitio.

Como resultado de los periodos cortos de investigación realizadas en el sitio, se ha podido develar que corresponde a un Centro Ceremonial Administrativo de ocupación Yschma, "la presencia de una tinaja y la impronta en el piso de otras, su ubicación es interesante, porque esta al centro de todos los recintos ceremoniales, restos domésticos (desperdicios de alimentos) asociados a la cerámica, con espacios de usos múltiples, que servían tanto como habitaciones, como para hacer trabajos muy especializados dedicados al uso de la elite que debe haber ocupado estos recintos", (Guillén, Informe final del proyecto arqueológico Huaca Huantille, 2012).

Las actividades de vinculación e inclusión con la población asentada en los alrededores del sitio arqueológico, quedaron inconclusas debido a la falta de presupuesto y predisposición por parte de las autoridades locales, por lo que la difusión de sus hallazgos y el reforzamiento de los lazos de identidad cultural, no obtuvieron los resultados que se esperaba. Además de las condiciones de conservación en la que se encontraba posterior a su recuperación, y en las cuales se centraron los primeros trabajos, esto debido a la elevada humedad del ambiente por su proximidad al mar, aunado a la basta evidencia de quema asociada a la basura moderna encontrada, siendo que varios de los bienes muebles presentan un regular estado de conservación, como manchas de ceniza, quema y ligeras exfoliaciones.

Actualmente, una parte de la población entrevistada manifiesta su preocupación por el uso social que se le viene dando a este patrimonio edificado; una demanda desatendida que se refleja en la ausencia de actividades que se puedan realizar: talleres, visitas guiadas, libre acceso al público, la falta de promoción y difusión, y sobre todo llama la atención cada vez la ausencia de seguridad permanente en el sitio como consecuencia del poco interés de las autoridades locales.

Esta propuesta, recomienda retomar actividades que impliquen la participación y el uso de este patrimonio, comenzando con la difusión de los principales hallazgos obtenidos en las intervenciones realizadas en el sitio, incluyendo a otros sectores sociales, (comerciantes y población aledaña) en las actividades que se desarrollen en el mismo; aprovechando el interés o apropiación que aún persiste de este patrimonio por parte de la comunidad del entorno, ello a consecuencia de los resultados obtenidos del sondeo y encuestas realizadas, que permitieron conocer qué tan importante era Huantille, y qué significado que tiene para ellos, los cual permitirá darles una interpretación adecuada del mismo, y finalmente continuar con la investigación y conservación que lo ameritan.

Es importante señalar, que este patrimonio arqueológico no puede verse separado del entorno urbano; específicamente, Lima Metropolitana presenta una inmensa muestra de este patrimonio que no puede pasar desapercibido por ser muestra y testimonio de sociedades milenarias, y que no han sido tomadas en cuenta dentro de una adecuada ,sólida y continua gestión por parte de las autoridades. Es en ese sentido que los lineamientos de gestión deberían ser empleados para el manejo y uso de este patrimonio edificado, se podrían retomar actividades con la ciudadanía de forma permanente, ya que cuenta con potencial arqueológico, turístico e infraestructura para el desarrollo de diversas actividades culturales y espacios de integración social que sirvan como escenarios para la difusión y consolidación de la identidad cultural y de su revaloración. 


\section{Reflexiones finales:}

La gestión del patrimonio cultural arqueológico en el entorno urbano de Lima, cobra importancia en este caso, superando un estado de ocupación ilegal, de abandono y dando paso a la investigación y a su puesta en valor, logrando y garantizando su salvaguarda, como un modelo recuperación patrimonial a través de la reubicación pacifica de sus ocupantes ilegales, en atención a un pedido de la misma población ubicada en el entorno.

En ese sentido, dicha recuperación debe buscar un acercamiento con la comunidad a través de su puesta en uso y disfrute social, para que desde su participación se garantice el desarrollo de actividades programadas a largo plazo y que garanticen su revaloración y la sostenibilidad en el tiempo.

\section{Bibliografía.}

Ampuero, G. (2017) Venturas y desventuras con el Patrimonio cultural (1953-1973). Editorial: Universidad de la Serena, Chile.

Ballart, J. \& Pericot, F. (1996). El valor del Patrimonio Histórico. Complutum Extra, 215-224

Boado, F. (2013). El patrimonio era otra cosa. Estudios Atacameños, Arqueología y Antropología Surandinas, (pp. 5-18) Atacama, Chile.

Consejo Internacional de Monumentos y Sitios (2008): Carta ICOMOS para interpretación y presentación de Sitios Patrimonio Cultural. 16² AG-Québec 2008.

Consejo Internacional de Museos (2007) La nueva versión de Estatutos ICOM aprobado en Asamblea General ICOM en Austria. http://icom.museum/

Criado, F. y González, M. (1995). La socialización del Patrimonio Arqueológico desde la perspectiva de la Arqueología del Paisaje. En S. d. Congreso, Actas del XXII Congreso Nacional de Arqueología (noviembre, 1993)

Espinosa, P. (2014). Arqueología: Catorce años de investigación en Maranga. La Arquitectura de Maranga en el Contexto del Núcleo Monumental Tardío del Valle bajo del río Rímac. Municipalidad Metropolitana de Lima. Patronato del Parque de las Leyendas. Lima-Perú

González, M. (2000). Memoria, Historia y Patrimonio: Hacia una concepción social de Patrimonio. Trabajos de Prehistoria Vol 57, № 2 (pp. 9-20).

Guillén, M. (2012) Descubrimientos arqueológicos del Intermedio Tardío en Huaca Huantille. Revista: Arqueológia y Sociedad №24, (pp. 371-391). Lima-Perú.

Guillén, M. (2007-2011). Informe Final Proyecto Arqueológico Huaca Huantille . Lima-Perú.

Guillén, M. (2011- 2012). Informe Final del Proyecto de investigación, conservación y puesta en Valor de a Huaca Huantille. Lima-Perú.

Hernández, F. (1998). El Museo como espacio de comunicación. Edit. Trea, S.L.Madrid-España:

Marcelo, M. (2007) La difusión del patrimonio. Actualización y debate. Dialnet-ladifusíon del patrimonio pp. 03, 04 y 05. http://www.revistadepatrimonio.es/revistas/numero1/difusion/estudios/articulo.php

Ladrón de Guevara, B. y Elizaga, J. (2009) Diagnóstico para la conservación de patrimonios culturales en uso activo: propuesta metodológica. Revista Conserva $\mathrm{N}^{\circ} 13-$ Madrid.

Lipe, W. (1983) Value and meaning in cultural resources (H. Cleere, cd.), Cambridge: 1-11.

Middendorf, E. (1984). Perú: Observaciones y estudios del País y sus Habitantes durante una permanencia de 25 años Tomo II: La Costa. Fondo Editorial de la Universidad Nacional Mayor de San Marcos.

Monsalve, L. (2011). Gestión del Patrimonio Cultural y Cooperación Internacional. Cuadernos de Cooperación para el Desarrollo № 6. Medellín - Colombia.

Ponceano, R. (2005) Patrimonio Arqueológico de Magdalena del Mar: La Huaca Huantille. Boletín Tukuy Rikuq. № 2. Lima-Perú 
devenir Vol. 7, Nº13, ENERO - JUNIO 2020, PP. 103-120 - EstudIOS I ISSN 2312-7562 | E-ISSN 2616-4949 UNIVERSIDAD NACIONAL DE INGENIERÍA, LIMA

doi: https://doi.org/10.21754/devenir.v7i13.927

Ravines, R. (1985) Inventario de Monumentos Arqueológicos del Perú (Lima Metropolitana) Primera aproximación. Municipalidad Metropolitana de Lima. Instituto Nacional de Cultura. Lima-Perú

Román, L. (2011) Una revisión teórica sobre la gestión cultural. Revista Digital de Gestión Cultural. Año 1. Número 1.

Tello, J. (1999) Cuadernos de Investigación del Archivo Tello. Museo de Arqueología y Antropología de la UNMSM. (pp. 82, 99, 102,109, 117). Lima-Perú

Zabala, M. (2006) Reflexiones teóricas sobre patrimonio, educación y museos. Revista de teoría y didáctica de las Ciencias Sociales Nº11 (pp.233-261). Mérida-Venezuela 